\title{
Percepção de discentes sobre o impacto da pandemia de COVID-19 nas atividades do Mestrado Profissional em Ensino em Saúde
}

\author{
Perception of students about the impact of the COVID-19 pandemic on the activities of \\ the Professional Master's in Health Education
}

\author{
Percepción de los estudiantes sobre el impacto de la pandemia COVID-19 en las \\ actividades de la Maestría Profesional en Educación para la Salud
}

\author{
Rosamaria Rodrigues Garcia ${ }^{1}$ \\ Raquel de Abreu Barbosa de Paula ${ }^{2}$ \\ Carlos Alexandre Felício Brito ${ }^{3}$
}

\section{Resumo}

Introdução: A pandemia de COVID-19 modificou abruptamente a sociedade e escancarou fragilidades dos sistemas políticos e econômicos. As atividades de ensino e pesquisa foram afetadas, obrigando instituições, docentes e discentes a reestruturarem suas ações. Objetivo: identificar a percepção de discentes matriculados no Programa de Mestrado Profissional em Ensino em Saúde da Universidade Municipal de São Caetano do Sul, sobre efeitos da pandemia nas atividades acadêmicas e de pesquisa. Método: estudo quali-quantitativo, transversal, descritivo, sendo aplicado questionário semiestruturado pelo Google Forms ${ }^{\circledR}$. Foi utilizada Análise de Conteúdo Automatizada, com o software livre Iramuteq ${ }^{\circledR}$ para tratamento dos dados. Resultados: foram recebidas 26 respostas, 88,5\% dos participantes estão envolvidos no enfrentamento da pandemia. A qualidade do corpus foi considerada quase adequada (64,71\% de aproveitamento). Emergiram 685 ocorrências, sendo 180 formas ativas (palavras distintas) e 58 suplementares, formando único conjunto. O conteúdo foi categorizado em 3 Classes (Clusters), a partir da Classe Hierárquica Descendente. As distribuições de segmentos de textos foram distintas. A Classe 2 foi a maior, com $39,40 \%$ dos segmentos de textos, seguida da Classe $1(33,3 \%)$ e da Classe $3(27,27 \%)$. Considerações Finais: de acordo com a percepção dos participantes, a pandemia de COVID-19 provocou alterações nas atividades acadêmicas e de pesquisa. As aulas presenciais foram substituídas pelo ensino remoto. No entanto, as atividades de pesquisa foram afetadas, principalmente quanto à coleta de dados e continuidade da pesquisa, sendo necessárias adaptações. Notou-se o efeito psíquico do isolamento social e mudança no estilo de vida, bem como no convívio com colegas nas atividades acadêmicas e de orientação. Novos estudos podem ser realizados, visando identificar efeitos longitudinais da pandemia nas atividades acadêmicas e de pesquisa.

\footnotetext{
${ }^{1}$ Fisioterapeuta, Doutora em Saúde Pública pela Universidade de São Paulo, Docente do Programa de Mestrado Profissional Inovação no Ensino Superior em Saúde da Universidade Municipal de São Caetano do Sul, São Caetano do Sul, Brasil. E-mail: rosamaria.garcia@online.uscs.edu.br ORCID: https://orcid.org/0000-0001-9454$\underline{6810}$

${ }^{2}$ Enfermeira, Mestre em Ensino em Saúde pela Universidade Municipal de São Caetano do Sul, São Caetano do Sul, Brasil. E-mail: pesquisa.raquel@gmail.com ORCID: https://orcid.org/0000-0003-0515-1679

${ }^{3}$ Educador Físico, Doutor em Educação Física pela Universidade Estadual de Campinas, Gestor e Docente do Programa de Mestrado Profissional Inovação no Ensino Superior em Saúde da Universidade Municipal de São Caetano do Sul, São Caetano do Sul, Brasil. E-mail: carlos.brito@online.uscs.edu.br ORCID: https://orcid.org/0000-0002-0060-8644
} 
Palavras-chave: mestrado profissional em saúde; ensino remoto; pesquisa científica; análise de conteúdo automatizada; COVID-19

\section{Abstract:}

Introduction: The COVID-19 pandemic abruptly changed society and opened up weaknesses in political and economic systems. Teaching and research activities were affected, forcing institutions, teachers and students to restructure their actions. Objective: to identify the perception of students enrolled in the Professional Master's Program in Health Education at Municipal University of São Caetano do Sul about the effects of the pandemic on academic and research activities. Method: qualitative, quantitative, cross-sectional, descriptive study, using a semi-structured questionnaire by Google Forms ${ }^{\circledR}$. Automated Content Analysis was used, with the free software Iramuteq ${ }^{\circledR}$ for data treatment. Results: 26 responses were received, $88.5 \%$ of the participants are involved in facing the pandemic. The quality of the corpus was considered almost adequate ( $64.71 \%$ of use). 685 occurrences emerged, with 180 active forms (distinct words) and 58 supplementary forms, forming a single set. The content was categorized into 3 Clusters, based on the Descending Hierarchical Class. The distributions of text segments were different. Cluster 2 was the largest, with $39.40 \%$ of the text segments, followed by Cluster 1 (33.3\%) and Cluster 3 (27.27\%). Final Considerations: according to the participants' perception, the COVID-19 pandemic caused changes in academic and research activities. Face-to-face classes were replaced by remote teaching. However, research activities were affected, mainly regarding data collection and research continuity, requiring adaptations. It was noted the psychic effect of social isolation and change in lifestyle, as well as in living with colleagues in academic and orientation activities. New studies can be carried out, aiming to identify the longitudinal effects of the pandemic in academic and research activities.

Keywords: professional master's in health; remote teaching; scientific research; automated content analysis; COVID-19

\section{Resumen:}

Introducción: La pandemia de COVID-19 cambió abruptamente la sociedad y abrió debilidades en los sistemas políticos y económicos. Las actividades de docencia e investigación se vieron afectadas, lo que obligó a las instituciones, docentes y estudiantes a reestructurar sus acciones. Objetivo: identificar la percepción de los estudiantes matriculados en el Programa de Maestría Profesional en Educación para la Salud de la Universidad Municipal de São Caetano do Sul sobre los efectos de la pandemia en las actividades académicas y de investigación. Método: estudio cualitativo, cuantitativo, transversal, descriptivo, mediante cuestionario semiestructurado de Google Forms®. Se utilizó Análisis de Contenido Automatizado, con el software gratuito Iramuteq ${ }^{\circledR}$ para el tratamiento de datos. Resultados: se recibieron 26 respuestas, el 88,5\% de los participantes están involucrados en el enfrentamiento a la pandemia. La calidad del corpus se consideró casi adecuada $(64,71 \%$ de uso). Surgieron 685 ocurrencias, con 180 formas activas (palabras distintas) y 58 formas complementarias, formando un solo conjunto. El contenido se clasificó en 3 clases (grupos), según la clase jerárquica descendente. Las distribuciones de los segmentos de texto fueron diferentes. La clase 2 fue la más grande, con el 39,40\% de los segmentos de texto, seguida de la clase $1(33,3 \%)$ y la clase $3(27,27 \%)$. Consideraciones finales: según la percepción de los 


\section{OO DEVIR EDUCAÇÃO}

ISSN: 2526-849X

participantes, la pandemia COVID-19 provocó cambios en las actividades académicas y de investigación. Las clases presenciales fueron reemplazadas por la enseñanza a distancia. Sin embargo, las actividades de investigación se vieron afectadas, principalmente en lo que respecta a la recopilación de datos y la continuidad de la investigación, lo que requirió adaptaciones. Se notó el efecto psíquico del aislamiento social y el cambio de estilo de vida, así como al convivir con colegas en actividades académicas y de orientación. Se pueden realizar nuevos estudios, con el objetivo de identificar los efectos longitudinales de la pandemia en las actividades académicas y de investigación.

Palabras-cláve: maestría profesional en salud; enseñanza a distancia; investigación científica; análisis de contenido automatizado; COVID-19

Introdução

Nos últimos anos, o persistente subfinanciamento do Sistema Único de Saúde (SUS), em parte como efeito deletério das austeras políticas econômicas orçamentárias, como a Lei de Responsabilidade Fiscal e a Emenda Constitucional 95 (definindo Teto de Gastos e drenando recursos orçamentários), vem minguando continuamente a capacidade operacional deste sistema. Dentre outros elementos que contribuíram para este estrangulamento e descrédito, encontram-se a extinção da CPMF (Contribuição Provisória sobre a Movimentação ou Transmissão de Valores e de Créditos e Direitos de Natureza Financeira), a permanência da DRU (Desvinculação de Receitas da União) e insistente atuação midiática contra a prestação de serviços ofertada pelo SUS (FLEURY, 2021).

Frente a um cenário de recursos financeiros insuficientes, com um crescente aumento de gastos em saúde e o envelhecimento da população brasileira, verifica-se cada vez mais a necessidade de reorganização do modelo assistencial que demanda a incorporação de novas tecnologias educacionais, que proporcionem a formação de profissionais de saúde com foco na integralidade do cuidado, para contrapor um modelo vigente na formação, orientado por conteúdos, que se reflete na fragmentação do cuidado.

No campo educacional, essas necessidades se expressam por meio da expansão das Escolas Médicas, com a Lei dos Mais Médicos e nas alterações propostas pelas Diretrizes Curriculares Nacionais (DCN) das Graduações de Saúde, que destacam a formação de profissionais baseados em perfis de egressos para atender os principais problemas de saúde da população com um olhar integral e humanizado (BRASIL, 2001; BRASIL, 2014). Para tanto, as DCN destacam a necessidade de maior carga horária na formação em contextos de prática, 
principalmente na Atenção Primária à Saúde e a adoção de metodologias ativas de ensino aprendizagem, com base em um currículo integrado.

O programa de Mestrado Profissional de Inovação no Ensino Superior em Saúde (MPIESS) da Universidade Municipal de São Caetano do Sul visa contribuir para a ampliação do acesso à saúde com qualidade, por meio da formação de profissionais envolvidos nas graduações da área da saúde, com a produção e disseminação de conhecimento aplicável e implantação de projetos inovadores, programas e políticas públicas, derivados dos artefatos e produções técnicas derivados das dissertações (USCS, 2017).

O programa destina-se aos docentes e profissionais de saúde atuantes nas atividades de ensino no âmbito dos serviços de saúde, especialmente no Sistema Único de Saúde (SUS), em suas diversas modalidades de atenção.

A Área de Concentração do MPIESS é "Inovações Educacionais em Saúde orientadas pela integralidade do cuidado" e pertence à área de Ensino, subárea temática de Ensino em Saúde. A vocação dessa área está relacionada à necessidade de formação de profissionais qualificados para o sistema de saúde brasileiro, por meio da elaboração de currículos integrados e do planejamento e alinhamento entre as instituições formadoras de profissionais de saúde e os profissionais envolvidos no ensino, atuantes nas redes de atenção à saúde, com o objetivo de ampliar o acesso ao cuidado com qualidade e segurança. Nesse sentido, esperase produção de conhecimentos e intervenções na formação de profissionais em graduações e pós-graduações em saúde (USCS, 2017).

O programa possui duas linhas de pesquisa, a saber: 1) Currículo Integrado em Saúde, que visa proporcionar o desenvolvimento de capacidades de elaborar, implantar e avaliar ações educacionais no ensino superior em saúde. Propõe uma abordagem inovadora para a construção de currículos integrados, com metodologias ativas de ensino aprendizagem, estratégias de aprendizagem e sistemas de avaliação; 2) Ensino em saúde nos contextos de prática do SUS, que tem por objetivo o desenvolvimento de capacidades de elaborar, implantar e avaliar projetos e ações educacionais nos contextos de prática do ensino em saúde, com a introdução de estratégias educacionais nos serviços de saúde que proporcionem integração entre universidade e rede de atenção, de forma a proporcionar melhorias tanto na formação profissional dos cursos graduação quanto na atenção à saúde da população (USCS, 2017). 
A pandemia da COVID-19 modificou abruptamente a sociedade contemporânea e escancarou as fragilidades dos sistemas políticos e econômicos vigentes nos diversos países do mundo. A propagação da nova classe do coronavírus, denominada SARS-CoV-2, abalou as estruturas sociais e assolou desprevenida a população mundial, desde o final de 2019 (WANG et al., 2020).

A principal medida amplamente adotada no mundo e recomendada pelas autoridades sanitárias para conter a disseminação da pandemia foi o isolamento/distanciamento social (SPÓSITO; GUIMARÃES, 2020; SANTOS, BRANDÃO \& ARAUJO, 2020).

A maioria das cidades brasileiras sofreu drásticas transformações: as escolas e universidades foram fechadas; apenas os estabelecimentos comerciais essenciais, que prestam serviços de alimentação humana e animal e serviços de saúde permaneceram abertos; as aglomerações foram proibidas; foi estabelecido o uso obrigatório de máscara na rua e nos estabelecimentos; foi recomendado o distanciamento mínimo e a aferição de temperatura; alguns municípios implantaram barreiras sanitárias nas estradas para conter a disseminação da doença, entre outras medidas de contingência (OLIVEIRA, 2020; BEZERRA et al., 2020).

A pandemia de COVID-19 aumentou a demanda por vários serviços ofertados pelo SUS, tais como atendimento de urgência e emergência, internação, medicina diagnóstica, vigilância epidemiológica e sanitária, acompanhamento na atenção primária, notificação, monitoramento de casos suspeitos, estudos científicos, vacinação, entre outros. Para tanto, fez-se necessária a implantação de medidas emergenciais, incluindo a suplementação do financiamento, bem como fortalecimento dos processos de normatização, coordenação e pactuação (FLEURY, 2021).

Os indivíduos estão vivendo um cenário inusitado em suas vidas, com situações inéditas, que geram reações e alterações em diversos aspectos da estrutura e organização social, incluindo as atividades acadêmicas e de pesquisa. Torna-se fundamental investigar os efeitos positivos e negativos do isolamento social e da imposição de novos formatos de interação entre discentes e docentes e entre pesquisadores e sujeitos de pesquisa (VERCELLI, 2020).

O presente estudo tem por objetivo identificar a percepção de profissionais de saúde matriculados no Programa de Mestrado Profissional Inovação no Ensino Superior em Saúde, da Universidade Municipal de São Caetano do Sul, sobre os efeitos da pandemia de COVID19 nas atividades acadêmicas e de pesquisa, relacionadas ao curso. 
Metodologia da pesquisa

A pesquisa aqui discutida se propõe a compreender fenômenos e processos em estudo e não está centrada em comprovação de hipóteses ou em obtenção de resultados prédeterminados, estando focada nos significados. Neste sentido, caracteriza-se como qualiquantitativa, pois utiliza-se de ferramentas estatísticas para a análise de conteúdo.

Segundo Creswell e Creswell (2021), uma pesquisa pode ter mais características da abordagem qualitativa do que quantitativa, mas incorpora elementos de ambas, caso em que pode ser considerada quali-quantitativa. $\mathrm{O}$ estudo qualitativo, ao ser aprofundado, pode gerar questões quantitativas e vice-versa. Esta pesquisa, de abordagem predominantemente qualitativa, teve dados colhidos em forma de palavras e utilizou o método misto de pesquisa paralelo.

Segundo Thomas, Nelson e Silverman (2012), este tipo de método também pode ser denominado como concorrente, pois implica que os componentes qualitativos investigados, bem como os quantitativos ocorrem ao mesmo tempo e de forma independente.

A investigação se desenvolveu durante o ano de 2020, devido ao período de pandemia, especificamente ao COVID-19. Entretanto, só foi possível investigar após o ano referido, portanto os dados foram coletados no início de 2021.

A coleta de dados foi realizada por um questionário constituído por 2 questões abertas sobre as mudanças geradas pela pandemia nas atividades acadêmicas e outra relacionada às atividades desenvolvidas durante a pesquisa. O instrumento foi composto das seguintes questões: 1. Por favor, descreva as mudanças geradas pela pandemia de COVID-19 em suas atividades acadêmicas (disciplinas) do programa de mestrado 2. Por favor, descreva as mudanças geradas pela pandemia de COVID-19 em suas atividades de pesquisa (desenho do estudo, coleta, análise de resultados, conclusão do trabalho, entre outras).

E ainda por 3 questões objetivas da seguinte forma: 1. Ano de ingresso no mestrado; 2. Situação do curso de mestrado (Em andamento, Concluído, Trancamento, Desistência); 3. Durante a pandemia de COVID-19, escolha a alternativa que melhor descreve a sua atuação (não trabalhei diretamente com pacientes com COVID-19 ou com suspeita desta doença; trabalhei diretamente com pacientes com COVID-19 ou com suspeita desta doença; não trabalhei com pacientes com COVID-19, mas trabalhei com setores ou aspectos diretamente 
relacionados à pandemia (exemplo: análises clínicas, diagnóstico, indústria farmacêutica e de insumos, transporte de insumos/equipamentos; trabalhei na gestão de serviços de saúde direta ou indiretamente relacionados ao COVID-19; Outros).

Neste estudo foram incluídos 26 participantes, o critério de inclusão foi o de serem discentes do curso de Mestrado Profissional em Inovação no Ensino Superior em Saúde, na região do $\mathrm{ABC}$, da cidade de São Paulo, sendo 14 homens e 12 mulheres, profissionais da área de Ciências da Saúde.

Análise de Conteúdo Automatizada

Os dados da presente pesquisa foram analisados por meio da técnica denominada de Análise de Conteúdo Automatizada. Esta técnica é oriunda da Análise de Conteúdo (CHIZZOTI, 2006), entretanto incorpora as possibilidades tecnológicas no tratamento de dados, apoiados em software e programas estatísticos para inferência. (GRIMMER; STEWART 2013).

A Análise de Conteúdo procura interpretar as diferentes formas do conteúdo de um texto que se desenvolveu, adotando normas sistemáticas de extrair os significados temáticos (CHIZZOTI, 2006). No presente estudo, estes significados temáticos foram desprendidos do texto, quando os discentes descreveram sobre os efeitos que a pandemia de COVID-19 trouxe durante a realização de suas pesquisas. "A ideia central desta técnica de análise consiste em relacionar a frequência da citação de alguns temas, palavras ou ideias em um texto para medir o peso relativo atribuído a um determinado assunto pelo seu autor" (CHIZOTTI, 2006, p. 114).

Muito embora está técnica de análise possa ser realizada sem auxílio de instrumento tecnológico, hoje em dia é possível desenvolver utilizando-se de software que aceleram este processo, portanto corroborando no desenvolvimento da pesquisa. Neste sentido, surge a Análise de Conteúdo Automatizado (GRIMMER; STEWART 2013), que facilita o processo de significação temática.

Para a Análise de Conteúdo Automatizada foi utilizado o software Iramuteq (Interface de R pour les Analyses Multidimensionnelles de Textes et de Questionnaires) na sua Versão 0.7 Apha 2 (http://www.iramuteq.org/), que é um software de livre acesso e que usa como linguagem estatística o R (RATINAUD, 2009). 
Como descreve Ratinaud (2019), trata-se de um software que viabiliza diferentes tipos de análise de dados textuais, desde aquelas bem simples, como a lexicografia básica, que abrange sobretudo a lematização e o cálculo de frequência de palavras; até análises multivariadas como classificação hierárquica descendente de segmentos de texto, análise de correspondências e análises de similitude. Por meio desse software, a distribuição do vocabulário pode ser organizada de forma facilmente compreensível e visualmente clara, com representações gráficas pautadas nas análises utilizadas.

As análises podem ser realizadas tanto a partir de um grupo de textos a respeito de uma determinada temática (Denominada de Corpus textual), reunidos em um único arquivo de texto; como a partir de matrizes com indivíduos em linha e palavras em coluna, organizadas em planilhas, como é o caso dos bancos de dados construídos a partir de testes de evocações livres. No presente estudo, as análises foram realizadas a partir das transcrições das respostas ao instrumento de pesquisa, como um único arquivo de texto.

$\mathrm{O}$ arquivo de texto foi preparado da seguinte forma:

1. Preparação do Corpus Textual. Como aponta Ratinaud (2019), o Corpus Textual é construído pelo pesquisador. É o conjunto de textos que se pretende analisar.

2. Os textos são separados por linhas de comando, também chamadas de "linhas com asteriscos" ou metadados. No caso de entrevistas, p. ex., como cada uma delas é um texto, e eles necessariamente devem começar com uma linha de comando, esta linha informa o número de identificação do entrevistado (do produtor do texto que se segue) e algumas características (variáveis) que são importantes para o delineamento da pesquisa.

Em posse das respostas dos participantes, foi preparado o Corpus textual monotemático (Ver mais em Tutorial para uso do software http://www.iramuteq.org/documentation) para análise dos resultados. O anonimato dos participantes da pesquisa foi preservado quanto a sua identidade e características, portanto não há descrição nominal ou outra forma de identificação.

A preparação do Corpus Textual para a Análise de Conteúdo Automatizada, com o software Iramuteq, foi realizada da seguinte forma: 
1. Foram criadas as linhas de comando (Algoritmo), para cada variável analisada, de acordo com o manual do Iramuteq;

2. Foram organizadas as seguintes categorias de análise (Variáveis): i) Atividades Acadêmicas e ii) Atividades de Pesquisa;

3. O texto foi formatado e salvo de acordo com as normas específicas do software.

Em posse do Corpus textual, preparado na presente pesquisa, optou-se pela análise dos resultados utilizando-se Nuvem de palavras e o Método de Reinert.

Uma nuvem de palavras é uma representação visual, do tipo grafo ou infográfico, na qual cada palavra é representada com um tamanho proporcional à frequência com que ela aparece no texto ou no conjunto de dados. Para gerar a nuvem, se identifica o número de vezes que cada palavra aparece no texto e se faz a distribuição de forma artística.

No caso da nuvem de textos curtos, ela representa de modo sintético o que existe de comum e mais frequente nos dados, auxiliando a visualização e nos levando a indagar os porquês da repetição de termos.

O algoritmo criado por Reinert gera clusters (aglomerados) de categorias lexicais, sem interpretação e interferência prévia do pesquisador. Logo, será o pesquisador, diante do pressuposto teórico, que fará a interpretação, assim como as inferências necessárias para compreender o fenômeno observado.

A partir dos clusters gerados é possível categorizar o texto pela Classificação Hierárquica Descendente (CHD), bem como pela Análise Fatorial de Correspondência (AFC). Ao realizar a CHD existem três possibilidades de análise, porém em nosso caso, utilizamos a CHD Simples sobre Texto (ST), pois esta é recomendada quando se faz análise das respostas curtas a partir de questionários abertos. A significância da clusterização foi identificada pelo teste estatístico a partir da frequência da citação utilizando o cálculo do Qui-quadrado. O valor tabelado pelo método Reinert para este teste é de 3,8, com nível significativo estatístico de probabilidade correspondente ao valor de $\mathrm{p} \leq 0,05$.

Resultados

Após o envio do questionário, foram recebidas 26 respostas, de 14 homens e 12 mulheres. Dentre as particularidades do programa e dos participantes do presente estudo, vale ressaltar a presença de discentes advindos de outros municípios de São Paulo e outros estados, 
como Paraná e Minas Gerais. Os discentes são majoritariamente profissionais médicos (dezenove), que atuam na rede pública e/ou privada de atenção à saúde, em geral na esfera terciária, concomitante à função de docentes ou tutores de cursos de graduação em Medicina. Compõem o restante dos participantes uma fisioterapeuta, quatro enfermeiras, uma farmacêutica e um psicólogo.

Com relação ao ano de ingresso no programa, um respondente matriculou-se em 2018, vinte e um discentes ingressaram em 2019 e quatro respondentes ingressaram em 2020. O referido programa encontra-se em andamento para 84,6\% dos participantes, 11,5\% concluíram o curso e um discente desistiu do curso $(3,8 \%)$, lembrando que o referido programa teve sua primeira turma ingressando em 2018.

Considerando a atuação durante a pandemia, 61,6\% dos respondentes estão trabalhando diretamente com pacientes com COVID-19 ou com suspeita da doença, 15,4\% não estão trabalhando diretamente com pacientes com COVID-19, mas atuam com setores ou aspectos diretamente relacionados à pandemia (exemplo: análises clínicas, diagnóstico, indústria farmacêutica e de insumos, transporte de insumos/equipamentos), 11,5\% estão trabalhando na gestão de serviços de saúde direta ou indiretamente relacionados ao COVID19, e 11,5\% referiram que não atuam com pacientes com COVID.

Observa-se que $88,5 \%$ dos participantes estão envolvidos no enfrentamento da pandemia. Alguns profissionais foram realocados de seus postos de trabalho ou de suas especialidades médicas, para atuarem majoritariamente no atendimento às pacientes infectados. Por exemplo, diante do cancelamento das cirurgias eletivas nos principais equipamentos da rede pública e privada, parte da carga horária dos médicos cirurgiões foi alocada no atendimento direto dos pacientes com COVID-19. Houve casos em que médicos da Atenção Básica foram realocados para serviços de atenção terciária. Em outras situações, os discentes foram convocados a trabalharem em plantões extras para compensar a falta de recursos humanos nos serviços.

Serão apresentados a seguir os resultados da análise de conteúdo automatizada.

Uma primeira aproximação pode ser apreendida a partir dos excertos representados na Nuvem de Palavras (Figura 1), na qual podemos perceber que a palavra mais frequente evocada foi "Online", que está centralizada na nuvem quando comparada às palavras menos citadas, que se localizam em sua periferia, por exemplo, a palavra "Presencial". Neste caso, a representação foi criada a partir dos relatos dos discentes na presente pesquisa, com auxílio do 
software Iramuteq (RATINAUD, 2009). A nuvem permitiu perceber os significados lexicais relevantes aos sujeitos envolvidos na pesquisa, expondo seus conhecimentos e percepções sobre o tema.

A partir da figura 1, analisando as palavras mais frequentes, pode-se inferir que os discentes do curso MPIESS descreveram a necessidade de que a pesquisa seja realizada atentando-se ao novo normal, ou seja, de forma remota online, de certa forma que a pandemia trouxe dificuldades na sua coleta, bem como na aplicação do seu projeto de pesquisa idealizado para aquele momento, neste novo contexto.

Figura 1. Nuvem de palavras criada no software Iramuteq ${ }^{\circledR}$.

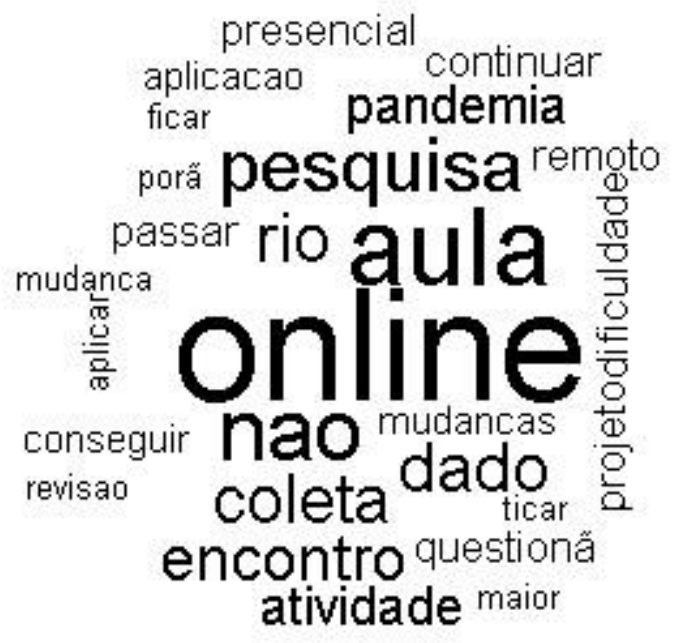

Fonte: Dos Autores (2021)

Como resultado da análise de conteúdo automatizada, a qualidade do corpus foi considerada quase adequada (grifo nosso), pois apresentou menos de $70 \%$ de aproveitamento, como indica o método de Reinert, o qual foi descrito na metodologia da pesquisa. O corpus foi separado em 88 segmentos de textos (ST), a partir das respostas e houve aproveitamento de 33 ST de 51 (64,71\%). Emergiram 685 ocorrências (palavras, formas ou vocábulos), sendo 180 formas ativas (palavras distintas) e 58 de forma suplementar, formando um único conjunto.

O conteúdo analisado foi categorizado em 3 Classes (Clusters), a partir da Classe Hierárquica Descendente (CHD) também obtida a partir do Método de Reinert. As distribuições de segmentos de textos foram distintas. A maior classe encontrada foi a Classe 


\section{QO DEVIR EDUCAÇÃO \\ ISSN: 2526-849X}

2, com cerca de 39,40\% dos segmentos de textos, quando comparado à Classe 3 com cerca de 27,27\%, assim como a Classe 1, com 33,33\% (Figura 2).

As características que mais se destacaram a partir da análise lexical, foram relativas às "Atividades Acadêmicas" (denominamos de: Classes 1 - atividades online e Classes 2 dificuldades), principalmente as relativas às "dificuldades" ( $\mathrm{X} 2=4,07 ; \mathrm{p}<0,043)$, em continuar e desenvolver a "pesquisa" $(\mathrm{X} 2=15,39$; $\mathrm{p}<0,0001)$. Por exemplo, nesta Classe 2 (Atividades Acadêmicas-dificuldades), pode-se observar o relato de alguns discentes para efeito de ilustração no que diz respeito às dificuldades. Ou seja, alguns discentes chegaram a relatar:

"[...] sintomas de esgotamento físico e mental". E continua, "[...] não consegui avançar mais na pesquisa e fiquei desestimulado a continuar", assim como outros discentes relataram "com a pandemia e as aulas online tive dificuldades no trabalho em casa, pois 2 filhos em casa sem escola e com muita energia, às vezes lenificavam o andamento e a evolução do trabalho, mas no final consegui adiantar bastante a pesquisa e a conclusão do trabalho".

As palavras em destaque (negrito) foram decodificadas pelo software, portanto podemos perceber na figura 2, que as mesmas aparecem descritas com maior frequência.

Figura 2. Classificação Hierárquica Descendente (CHD). Classes de segmentos de textos (Total de 3) extraídas a partir das respostas dos discentes do curso de pós-graduação. CHD construído a partir do software Iramuteq.

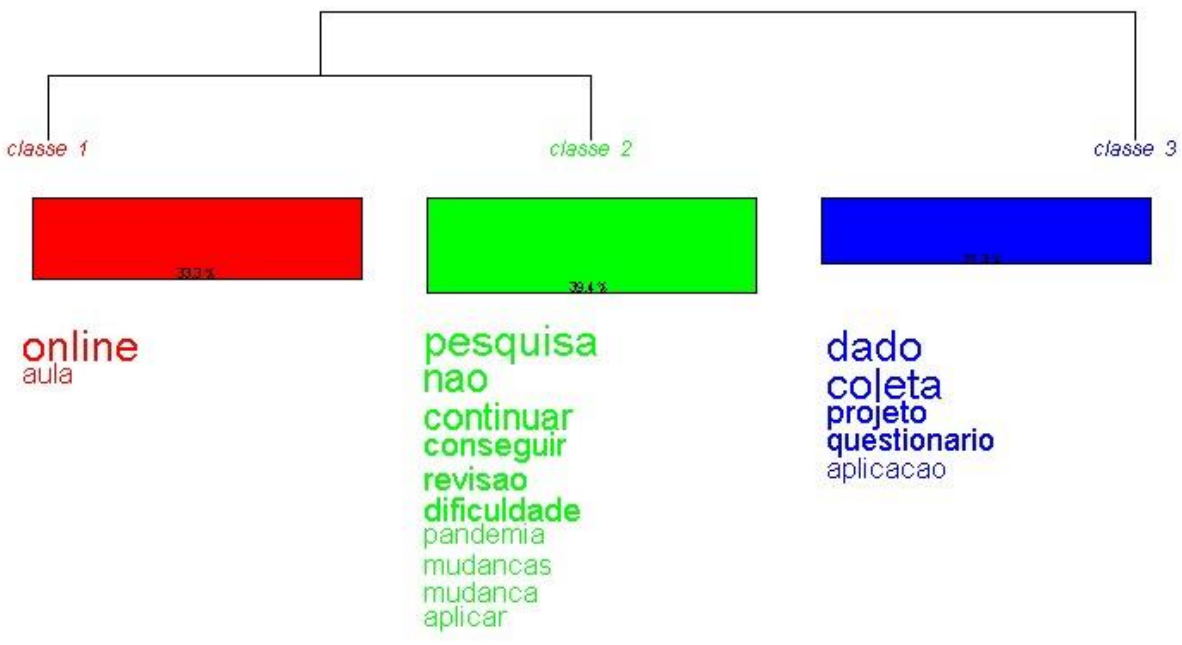

Fonte: Dos Autores (2021)

Mas, como podemos entender ou denominar cada Classe?

Revista Devir Educação, Lavras-MG. Edição Especial, p.10-28, Set./2021. 
Na figura 2, observa-se que foram geradas 3 Classes pela análise de conteúdo automatizada e essas se encontram divididas em duas ramificações do corpus total de análise, também chamados de subcorpus. As Classes 1 e 2 estão isoladas das demais devido às suas especificidades, que são diferentes das Classes 3. Assim sendo, são identificados dois subcorpus (A e B).

De acordo com as características do subcorpo A este foi denominado de "Atividades Acadêmicas-online". Não obstante, e apoiando-se em Arruda (2020), com objetivo de evidenciar a excepcionalidade da situação em tempos de COVID-19, nos traz à reflexão a questão do isolamento social e as ações a serem pensadas na Educação Remota Emergencial (ERE), assim como suas implicações nos diferentes níveis educacionais.

No que se refere ao Programa de Mestrado Profissional da universidade em que estão matriculados os participantes do presente estudo, todas as atividades acadêmicas passaram a ser realizadas remotamente, após convênio estabelecido entre a universidade e o Google ${ }$, por meio da aquisição do pacote Google Suit for Education ${ }^{\circledR}$. Foram criadas turmas no Google Classroom ${ }^{\circledR}$ e os discentes foram ensalados conforme o ano de ingresso no programa.

Disponibilizou-se treinamentos e tutorais para docentes e discentes e celeremente todas as atividades foram retomadas, a saber, disciplinas obrigatórias, disciplinas optativas, seminários de pesquisa, orientações de dissertações, cafés filosóficos, reuniões pedagógicas, capacitações docentes, eventos científicos, entre outros, corroborando o relato de Vercelli (2020), em que atividades semelhantes também foram implantadas em um programa de estrão profissional em Educação.

O calendário escolar apresentou discretas alterações, sem prejuízo na carga horária das disciplinas (idêntica à carga da modalidade presencial), inclusive mantendo o horário de início e de término das aulas, bem como houve a manutenção dos docentes que já ministravam as disciplinas do programa.

Outra medida adotada pela universidade foi a aquisição de assinaturas da plataforma Zoom®, exclusivas aos cursos de Stricto Sensu da universidade, que apresenta facilidades de uso e funções diferenciadas para criação de salas virtuais para atividades em grupo, conversas via chat, inserção de convidados externos etc.

Em termos quali-quantitativos podemos observar em seus relatos que este subcorpo A foi determinante aos discentes nesta época de pandemia, pois alguns relataram que, a 


\section{OO DEVIR EDUCAÇÃO \\ ISSN: 2526-849X}

"adaptação para o ensino online $(X 2=13,75 ; \mathrm{p}<0,0002)$, nunca tinha feito aulas $(\mathrm{X} 2=8,25 ; \mathrm{p}<0,004)$ nesse modelo, mas $[\ldots]$ foi bastante produtivo e inovador para mim". E ainda, outro discente relata que o "[...] questionário já seria aplicado via online, mas eu o apresentaria e distribuiria pessoalmente nas salas de aula. Com a pandemia, o questionário foi distribuído via aplicativo WhatsApp ${ }^{\circledR}$ e acho que acabou diminuindo o meu retorno das respostas", portanto relatando as implicações deste momento tão delicado.

Um dos pontos relevantes que Arruda (2020, p.258) considera, em relação ao isolamento social ocorrido em 2020, é que ele

"[...] promoveu transformações econômicas severas imediatas, com a parada obrigatória de inúmeros setores, modificou nossa relação com a arte, devido à ausência do compartilhamento presencial de experiências de fruição e, no caso da educação, promoveu desconstruções sob a forma como o ensino e a aprendizagem são vistos socialmente $[. .]$.$" .$

No caso particular do Ensino Superior, parece haver uma menor resistência na adesão à ERE por parte dos docentes, quando comparados aos docentes de outros níveis de ensino. Isto parece ser explicado, na perspectiva de Arruda (2020, p.266), por

"[...] atenderem pessoas adultas que não se encontram em processo de formação inicial que envolve o contato físico, a movimentação do corpo e a socialização nos seus mais diferentes níveis - como é o caso da educação dos jovens, da educação infantil, do ensino fundamental e médio [...].

A situação enfrentada ao longo do ano de 2020, introduziu na prática docente elementos novos que, por sua vez, exigiram uma ressignificação de todo o fazer pedagógico, o que inclui os gestos didáticos, mesmos os mais radicados e fundadores.

Na prática pedagógica, o docente se expressa por meios verbais e, também, por meios não verbais. Essas são as formas de expressão docente observáveis e que encaminham suas ações didáticas, atitudes e emoções. Pode-se dizer que são os seus gestos de trabalho, ou seja, os gestos didáticos, com os quais o docente exerce a docência. Nos sistemas semióticos dos docentes, usar determinados gestos ou optar por não usar gestos no ambiente da aula, 


\section{OD DEVIR EDUCAÇÃO \\ ISSN: 2526-849X}

introduzindo espaços de silêncio, são formas produtivas para ensinar e potencializar nos discentes a atividade de aprender (NASCIMENTO, 2011).

O subcorpo B, formado pelas Classes 2, que guardam semelhanças entre si, foi denominado de "Atividades Acadêmicas-dificuldades", pois se referem às consequências da pandemia devido ao isolamento social em função da COVID-19. Alguns discentes relataram estas dificuldades devido a

"mudança de metodologia de pesquisa em virtude de aplicação do método por mudanças que ocorreram inclusive na gestão de serviços", assim como alguns participantes da pesquisa relataram que "continua em pesquisa no computador", porém com "[...] maior dificuldade para acessar o professor", uma vez que "[...] agora apenas online"

Uma perspectiva relevante na pesquisa qualitativa é a utilização da Análise Fatorial de Correspondência (AFC). Foi possível realizar associação do texto entre as palavras, considerando a frequência de incidência de palavras e das classes formadas, representando-as em um plano cartesiano (Figura 3). Observa-se que as palavras de todas as classes se apresentam num segmento centralizado que se expande para pontos periféricos.

As Classes 1, 2 e 3, representadas pelas cores vermelho (Classe 1), verde (Classe 2) e azul (Classe 3), estão separadas entre os quadrantes. Isto posto, pode ser interpretado que, mesmo fazendo parte do subcorpus A e B, como descrito anteriormente, há diferenças entre elas em função da oposição que elas se encontram nos eixos cartesianos. A partir desta análise, ou seja, da AFC é possível supor que a Classes 3 explica cerca de 39,44\% da variância sobre o que denominamos de "Atividades Acadêmicas-pesquisa" (Fator 2, eixo “y”).

Figura 3. Representação gráfica 1x2 da Análise Fatorial de Correspondência (AFC) do Corpus Textual 


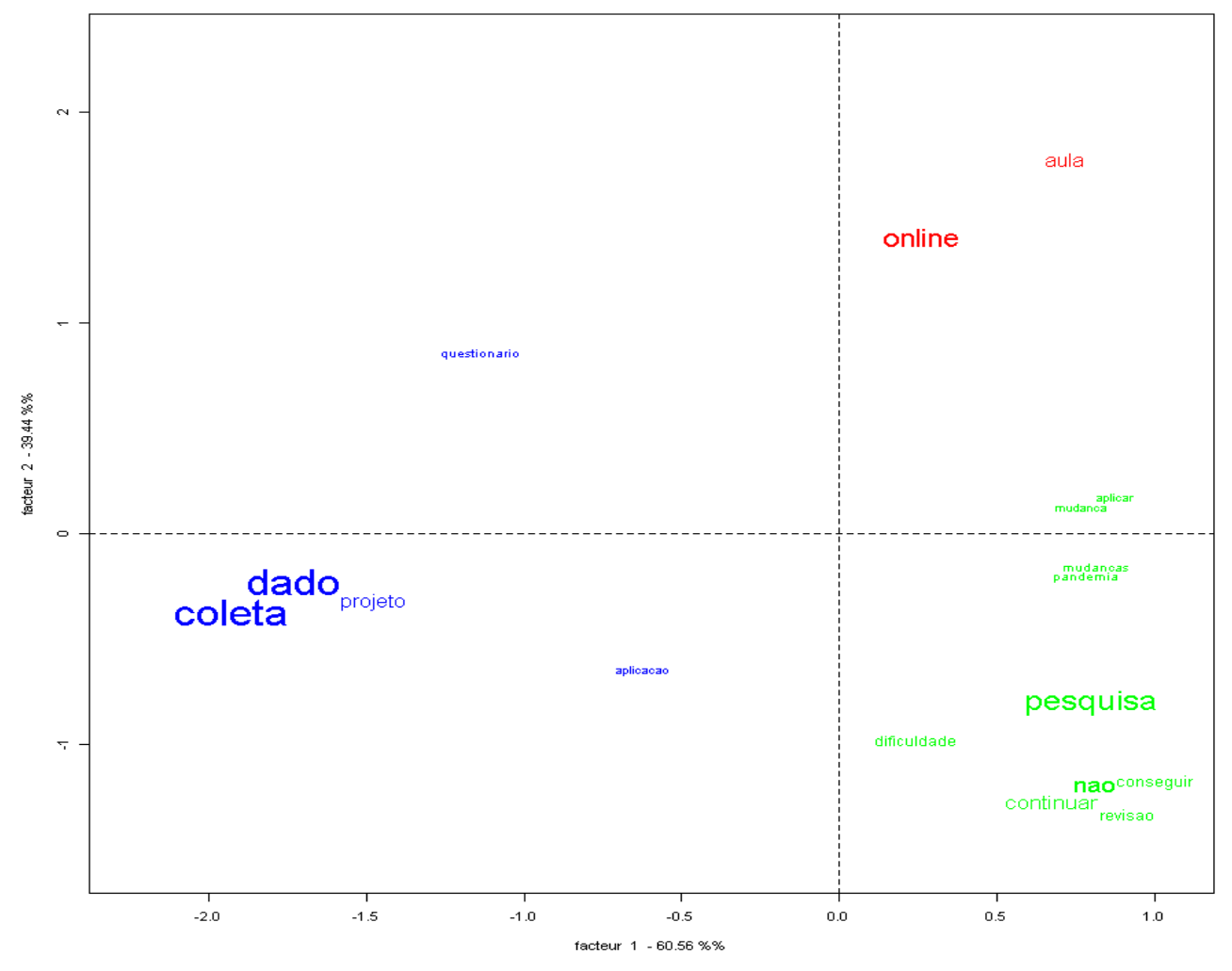

Fonte: Dos Autores (2021)

Portanto, parece que a AFC indica que os discentes percebem este processo de forma significativa neste momento de pandemia. Ressaltamos que as impressões percebidas, naquele momento, indicaram evidências negativas, as quais destacamos o

\footnotetext{
"impacto de diversas maneiras no projeto $(\mathrm{X} 2=8,26 ; \mathrm{p}<0,004)$ toda coleta $(\mathrm{X} 2=23,69 ; \mathrm{p}<0,0001)$ de $\operatorname{dados}(\mathrm{X} 2=23,69 ; \mathrm{p}<0,0001)$ [e nos] questionários, assim como na "[...] capacitação, pois tudo tem sido feito online através de ferramentas como Zoom ${ }^{\circledR}$ e Google Forms ${ }^{\circledR}$ ". Alguns discentes chegaram a relatar que houve atraso na coleta dos dados enfatizando que, "o COVID-19 atrasou o início da coleta de dados".
}

Portanto, podemos perceber as influências negativas que houve durante a pandemia no ano de 2020, especificamente nas atividades de pesquisa, quer sejam de natureza do método da pesquisa a ser utilizado, assim como das dificuldades percebidas devido ao isolamento social em época de COVID-19.

Considerações Finais 
De acordo com a percepção dos participantes, a pandemia de COVID-19 provocou alterações na operacionalização das atividades acadêmicas e de pesquisa do MPIESS. As aulas presenciais foram substituídas pelo ensino remoto, sem prejuízo no conteúdo programático e na carga horária. No entanto, as atividades de pesquisa foram afetadas, principalmente no que se refere à coleta de dados e continuidade da pesquisa, sendo necessárias adaptações nos instrumentos, na aplicação e em alguns casos no desenho de pesquisa.

Notou-se entre os participantes o efeito psíquico do isolamento social e da mudança no estilo de vida, bem como no convívio com os colegas nas atividades acadêmicas e de orientação. Novos estudos podem ser realizados, a partir de entrevistas em profundidade, visando identificar os efeitos longitudinais nas atividades acadêmicas e de pesquisa de profissionais de saúde matriculados em cursos Stricto Sensu, provocados pela pandemia de COVID-19.

\section{Referências}

ARRUDA, Eucidio Pimenta. Educação remota emergencial: elementos para políticas públicas na educação brasileira em tempos de Covid-19. EmRede Revista de Educação a Distância, Porto Alegre, v. 7, n. 1, p. 257-275, maio 2020.

BEZERRA, Anselmo César Vasconcelos; SILVA, Carlos Eduardo Menezes da; SOARES, Fernando Ramalho Gameleira; SILVA, José Alexandre Menezes da. Fatores associados ao comportamento da população durante o isolamento social na pandemia de COVID-19. Ciência \& Saúde Coletiva, Rio de Janeiro, v. 25, Supl. 1, p. 2411-2421, jun. 2020.

BRASIL. Ministério da Educação. Conselho Nacional de Educação. Câmara de Educação Superior. Parecer CNE/CES n. ${ }^{\circ} 1.133$, de 7 de agosto de 2001. Institui Diretrizes Curriculares Nacionais dos Cursos de Graduação em Enfermagem, Medicina e Nutrição. Diário Oficial da União: Seção 1E, Brasília, DF, p.131, 3 out. 2001.

BRASIL. Ministério da Educação. Conselho Nacional de Educação. Câmara de Educação Superior. Resolução CNE/CES n. ${ }^{\circ}$ 3, de 23 de junho de 2014. Institui Diretrizes Curriculares Nacionais do Curso de Graduação em Medicina e dá outras providências. Diário Oficial da União: Seção 1, Brasília, DF, p. 8-11, 23 jun. 2014.

CHIZZOTI, Antonio. Pesquisa qualitativa em ciências humanas e sociais. Petrópolis: Vozes, 2006. 
CRESWELL, John. W.; CRESWELL, J. David. Projeto de pesquisa: métodos qualitativo, quantitativo e misto. Porto Alegre: Penso, 2021.

FLEURY, Sonia. Políticas de isolamento na pandemia: confrontação federativa, disputas discursivas e consequências político-sanitárias. In: SANTOS, Alethele de Oliveira; LOPES, Luciana Tolêdo (Org.). Principais Elementos. Coleção Covid-19. Brasília: Conselho Nacional de Secretários de Saúde, 2021, v. 1, p. 110-125.

GRIMMER, Justin; STEWART, Brandon M.. Text as data: the promise and pitfalls of automatic content analysis methods for political texts. Political Analysis, v. 21, n. 3, p. 267297, jan. 2013.

NASCIMENTO, Elvira Lopes. A dupla semiotização dos objetos de ensino-aprendizagem: dos gestos didáticos fundadores aos gestos didáticos específicos. Signum: estudos da linguagem, Londrina, v. 14, n. 1, p. 421-445, jun. 2011.

OLIVEIRA, Victor Hugo Nedel. Juventudes, escola e cidade na pandemia da Covid-19. Boletim de Conjuntura (BOCA), Boa Vista, v. 4, n. 10, p. 115-122, out. 2020.

RATINAUD, Pierre. IRAMUTEQ: Interface de R pour les Analyses Multidimensionnelles de Textes et de Questionnaires. Computer software. Austria, 2009. Disponível em: http://www.iramuteq.org. Acesso em 29 jan. 2021.

SANTOS, Stephany da Silva; BRANDÃO, Gisetti Corina Gomes; ARAÚJO, Kleane Maria da Fonseca Azevedo. Social isolation: a look health elderly mental during the COVID-19 pandemic. Research, Society and Development. Vargem Grande Paulista, v. 9, n. 7, p. 1-15, jul. 2020.

SPOSITO, Maria Encarnação Beltrão; GUIMARÃES, Raul Borges. Por que a circulação de pessoas tem peso na difusão da pandemia. Portal Eletrônico da UNESP. São Paulo, SP: Universidade Estadual Paulista, 2020. Disponível em: https://www2.unesp.br/portal\#!/noticia/35626/por-que-a-circulacao-de-pessoas-tem-peso-nadifusao-da-pandemia. Acesso em 02 dez 2020.

THOMAS, Jerry R.; NELSON, Jack K.; SILVERMAN, Stephen J.. Métodos de pesquisa em atividade física. Porto Alegre: Artmed, 2012.

USCS - UNIVERSIDADE MUNICIPAL DE SÃO CAETANO DO SUL. Mestrado Profissional Inovação no Ensino Superior em Saúde. São Caetano do Sul, SP: Universidade Municipal de São Caetano do Sul, 2017. Disponível em https://www.uscs.edu.br/pos-stricto-sensu/ppgs/mestrado-profissional-em-saude. Acesso em 31 jan 2021.

VERCELLI, Lígia de Carvalho Abões. Aulas remotas em tempos de Covid-19: a percepção de discentes de um programa de mestrado profissional em educação. Revista @ mbienteeducação, São Paulo, v. 13, n. 2, p. 47-60, mai/ago. 2020. 


\section{QO DEVIR EDUCAÇÃO}

ISSN: 2526-849X

WANG, Chang; HORBY, Peter W.; HAYDEN, Frederick G.; GAO, George F.. A novel coronavirus outbreak of global health concern. The Lancet, London, v. 395, n. 10223, p. 470473, feb. 2020.

Recebido em: 26/02/21

Aprovado em: 07/05/21

Revista Devir Educação, Lavras-MG. Edição Especial, p.10-28, Set./2021. 